\title{
Effectiveness and Safety of Bedaquiline-containing Regimens for Treatment on Patients With Refractory Rifampicin/multidrug- resistant Tuberculosis: A Retrospective Cohort Study in East China
}

\author{
Shao-Jun Zhang \\ Tongji University \\ Yan Yang \\ Tongji University \\ Wen-Wen Sun \\ Tongji University \\ Zhong-Shun Zhang \\ Tongji University \\ He-ping Xiao \\ Tongji University \\ Yu-Ping Li \\ Tongji University \\ Zhe-Min Zhang \\ Tongji University \\ Lin Fan ( $\nabla$ fanlinsj@163.com ) \\ Tongji University
}

\section{Research Article}

Keywords: bedaquiline, multidrug-resistant, rifampicin-resistant, refractory tuberculosis

Posted Date: August 30th, 2021

DOI: https://doi.org/10.21203/rs.3.rs-833736/v1

License: () (i) This work is licensed under a Creative Commons Attribution 4.0 International License. Read Full License 


\section{Abstract}

Objective: To compare the effectiveness and safety of bedaquiline-containing and bedaquiline-free regimens for treatment of patients with refractory rifampicin-resistant/multidrug-resistant tuberculosis (RR/MDR-TB).

Methods: Patients with refractory RR/MDR-TB receiving bedaquiline-containing regimens (bedaquiline group, $\mathrm{n}=102$ ) and bedaquiline-free regimens (non-bedaquiline group, $n=100$ ) were included in this retrospective historical control study across East China. The culture conversion, end-of-treatment outcomes, cavity closing rate, and adverse events (AEs) were compared between the two groups. Univariate and multivariate analyses were performed to identify independent predictors of treatment success and culture reversion.

Results: The baseline characteristics of the patients were well balanced between the two groups. The culture conversion rates in the bedaquiline group at month $3(89.2 \%$ vs. $66.0 \%)$, month 6 (90.2\% vs. $72.0 \%)$, month $9(91.2 \%$ vs. $66.0 \%)$, and month 12 (94.1\% vs. $65.0 \%$ ) were all significantly higher than those in the non-bedaquiline group (all $p<0.001$ ). Similar results were observed in the cavity closing rate at month $9(19.6 \%$ vs. $8.0 \%, p=0.017)$ and month $12(39.2 \%$ vs. $15.0 \%, p<0.001)$. Patients receiving bedaquilinecontaining regimens had more treatment success than those receiving bedaquiline-free regimens $(p<0.001$; cure rate, $69.6 \%$ vs. $45.0 \%$; complete the treatment, $22.5 \%$ vs. $18.0 \%$; treatment success, $92.2 \%$ vs. $63.0 \%$ ). The use of bedaquiline was identified as an independent predictor of both treatment success (OR=7.356, 95\% Cl: $2.920-18.530, p<0.001)$ and culture reversion $(\mathrm{OR}=0.124,95 \%$ Cl: $0.035-0.452, p<0.001$ ). AEs were similarly reported in $26.5 \%$ of patients in the bedaquiline group and $19.0 \%$ in the nonbedaquiline group $(p=0.206)$.

Conclusions: Bedaquiline-containing regimens resulted in better treatment outcomes and similar safety relative to bedaquiline-free regimens for patients with refractory pulmonary RR/MDR-TB.

\section{Introduction}

Drug-resistant tuberculosis (DR-TB) remains a public health burden and represents a serious threat to patients, communities and health care services [1]. The World Health Organization (WHO) estimated that there were 465000 incident cases of rifampicinresistant tuberculosis (RR-TB) in 2019, and 78\% had multidrug-resistant tuberculosis (MDR-TB) [2]. However, the treatment outcomes of RR/MDR-TB remain poor, with a treatment success rate of 57\% from a global TB report in 2020 [2]. Therefore, novel and effective anti-TB agents are urgently needed to improve the treatment outcomes of RR/MDR-TB.

Bedaquiline is a new anti-TB agent that has been confirmed to improve treatment outcomes and culture conversion rates when added to conventional MDR-TB treatment regimens [3, 4]. Based on the above evidence, bedaquiline has been recently recommended by the WHO as an initial drug for use in all-oral MDR-TB treatment regimens [5]. Nevertheless, for patients with refractory MDR-TB (e.g., intolerance, multiple drug resistance, or discontinued treatment due to side effects), treatment is extremely complex [6]. In this group of patients, it is challenging to compose treatment regimens that contain at least four likely effective agents [7]. Until now, definite treatment modalities have not been identified for patients with refractory RR/MDR-TB. In addition, whether bedaquiline has a clear-cut benefit in this group of patients remains unclear. In China, bedaquiline was introduced into routine care for the treatment of MDR-TB in 2018 [8]. Therefore, this retrospective cohort study analyzed the treatment outcomes of patients with refractory RR/MDR-TB receiving bedaquiline-containing regimens from August 2018 to August 2020 and compared them with those receiving bedaquiline-free regimens between August 2016 and July 2018.

\section{Materials And Methods}

\section{Study design and patients}

The retrospective cohort study across East China (Shanghai, Anhui, Zhejiang, Jiangxi and Jiangsu province) was designed to analyze the treatment outcomes of patients with refractory RR/MDR-TB receiving bedaquiline-containing regimens during August 2018-August 2020 (bedaquiline group), and compared them with those receiving bedaquiline-free regimens between August 2016 and July 2018 (non-bedaquiline group). Ethics approval was obtained from the Institutional Review Board of Shanghai Pulmonary Hospital (number: 114JH), and informed consent was obtained from each participant. 
Refractory MDR-TB was defined as a patient infected with Mycobacterium tuberculosis (MTB) that was resistance to both rifampicin and isoniazid, a patient for whom an effective regimen (fewer than 4 effective agents due to intolerance or multiple drug resistances) cannot be developed, or a patient for whom treatment was discontinued because of adverse events (AEs). The inclusion criteria of patients were as follows: 1) patients with RR/MDR-TB confirmed by sputum culture and drug susceptibility tests (DST); 2) patients for whom effective regimens (fewer than 4 effective agents due to intolerance, discontinuation of a secondline drug regimen due to AEs or multiple drug resistances) could not be developed; and 3) patients who consented to participate in the study and signed the consent form. Patients with severe heart, liver, lung or kidney dysfunction, malignant tumors, bedaquiline allergy, positive culture identified as non-tuberculous mycobacteria, Fridericia-corrected QT (QTcF) interval > 450 ms, or significant electrocardiograph abnormalities at screening were excluded from the study.

\section{Treatment regimens}

The treatment regimens of all patients were uniformly formulated by specialists group in Shanghai Pulmonary Hospital and managed by the Shanghai Municipal Center for Disease Control \& Prevention. According to WHO guidelines, individualized treatment regimens were designed for patients based on their previous histories of anti-TB treatment and DST results. The total treatment duration was 18-20 months.

In the non-bedaquiline group, patients with RR/MDR-TB initially started treatment with a standardized regimen of at least five antiTB drugs in the intensive phase, including fluroquinolone (levofloxacin or moxifloxacin), Second-line injectables (capreomycin or amikacin), cycloserine, protionamide (ethambutol), pyrazinamide, and clofazimine (linezolid or para-aminosalicylic).

Patients in the bedaquiline group received bedaquiline-containing regimens. Bedaquiline was administered for 24 weeks (with a loading dose of $400 \mathrm{mg}$ once a day for the initial 2 weeks, followed by $200 \mathrm{mg}$ three times a week for the remaining 22 weeks). Background regimens consisted of at least 4 anti-TB drugs in the intensive phase, including fluroquinolone (levofloxacin or moxifloxacin), injectable agents (capreomycin or amikacin), cycloserine, protionamide, ethambutol, pyrazinamide, clofazimine, linezolid, or para-aminosalicylic.

\section{Treatment evaluation}

Information on the patients' demographic characteristics, background regimens, TB treatment history, laboratory test results, sputum culture conversion, end-of-treatment outcomes, radiological findings and AEs was collected.

The primary effectiveness outcomes of interest were culture conversion and end-of-treatment outcomes. Culture conversion was defined as two consecutive negative results received at least 30 days apart in a patient with positive sputum culture results at baseline. The sputum culture conversion status was compared between the two groups at 3, 6, 9, and 12 months following treatment initiation. Culture reversion was defined as two consecutive positive sputum cultures at least 30 days apart after initial culture conversion [9]. The end-of-treatment outcomes were assigned according to WHO definitions [10], including treatment success (cure or treatment completion), treatment failure, loss to follow-up, and death.

Imaging evaluation was performed every 3 months during the treatment duration. The cavity closing rate was calculated by dividing the number of patients who had cavity closure by the number of patients found to have cavitary lesions at baseline in each group.

According to the TB treatment history, patients could be classified into new cases (patients who had never been treated for TB or had taken anti-TB medicines for less than 1 month) and previously treated cases (patients who had received anti-TB medicine more than 1 month in the past). In addition, previously treated cases were further divided into patients with a one-time TB treatment history, patients with a two-time TB treatment history, and patients with a three-time TB treatment history. Patients with a one-time TB treatment history were those who had previously been treated one time and whose treatment failed or relapsed. Patients with a two-time TB treatment history were those who had previously been treated twice and whose treatment failed or relapsed at the end of their most recent course of treatment. Patients with a three-time TB treatment history were those who had previously been treated three times and whose treatment failed or relapsed at the end of their most recent course of treatment.

AEs of interest were mainly evaluated by laboratory-monitored parameters (leucopenia, liver function, renal function, etc.) and electrocardiography (QTcF interval prolongation). Significant QTcF interval prolongation was defined as any QTcF interval value $\geq$ 
500 ms or any increase in QTcF interval value $>60 \mathrm{~ms}$ from the baseline [11].

\section{Statistical analysis}

Quantitative variables were expressed as the means \pm standard deviations (SD) or median interquartile range (IQR) values and compared using Student's t-test. Qualitative data were expressed as numbers and percentages and evaluated using the chi-square test. The median times of culture conversion and reversion were estimated using the Kaplan-Meier method and compared across different regimens using a log-rank test. Logistic regression was used to identify the independent predictors associated with end-oftreatment success and culture reversion. Statistical analyses were performed using SPSS version 26.0 (SPSS Institute. IL. USA), and statistical significance was set at $p<0.05$.

\section{Results}

\section{Patient characteristics}

Overall, 202 eligible patients with refractory RR/MDR-TB were included in this retrospective study. Among the 202 patients, 102 were treated with bedaquiline-containing regimens (bedaquiline group), and they had a median age of 37.0 years (IQR, 28.0-52.0). The other 100 patients (median age, 43.5 years; IQR, 29.0-54.0) were treated with bedaquiline-free regimens (non-bedaquiline group). The baseline characteristics of the included patients are listed in Table 1. The two groups were generally well balanced for age, sex, cavitary disease, extrapulmonary TB, diabetes, TB treatment history and drug resistance type (all $p>0.05$ ).

\section{Background regimens}

The background drugs included in the treatment regimens of patients from the two groups are presented in Fig. 1. In the bedaquiline group, the most frequently used background drugs included pyrazinamide (102/102, 100\%), linezolid (92/102, 90.2\%), clofazimine (92/102, 90.2\%), cycloserine (77/102, 75.5\%), para-aminosalicylic acid (72/102, 70.5\%) and protionamide (60/102, $58.8 \%)$. Meanwhile, cycloserine (90/100, 90.0\%), capreomycin (81/100, 81.0\%), protionamide $(72 / 100,72.0 \%)$ and clofazimine $(51 / 100,51.0 \%)$ were the major components in non-bedaquiline group.

\section{Sputum culture conversion}

Among the 102 patients with culture positivity at baseline in the bedaquiline group, culture conversion rates of 89.2\% (91/102), 90.2\% (92/102), 91.2\% (93/102) and 94.1\% (96/102) were reported at months 3, 6, 9, and 12, respectively. Of the 100 patients with culture positivity at baseline in the non-bedaquiline group, 66.0\% (66/100), 72.0\% (72/100), 66.0\% (66/100), and 65.0\% (65/100) achieved culture conversion at months $3,6,9$, and 12, respectively. Significant differences were found at all time points between the two groups (all $p<0.001$ ). In addition, the median time of culture conversion in the bedaquiline group was 3.0 months (IQR, 3.0-3.0), which was significantly lower than that in the non-bedaquiline group (5.8 months [IQR, 3.0-12.0]; $p<0.001 ;$ Fig. $2 \mathrm{~A}$ ). In addition, patients in the bedaquiline group had a lower rate of reversion to positive culture than those in the non-bedaquiline group (2.9\% vs. $18.0 \%, p<0.001$; Fig. 2 B) .

Subgroup analyses of the drug resistance type or TB treatment history on the 6-month culture conversion rate of the two groups is shown in Table 2. The 6-month culture conversion rate among patients with MDR-TB in the bedaquiline group was significantly higher than that for patients in the non-bedaquiline group ( $87.8 \%$ vs. $73.5 \%, p<0.05)$. However, significant differences were not found in the 6-month culture conversion rate of patients with RR-TB or XDR-TB between the two groups (all $p>0.05$ ). In addition, patients with $\mathrm{a} \geq$ three-time TB treatment history had a higher 6-month culture conversion rate in the bedaquiline group than in the non-bedaquiline group $(86.4 \%$ vs. $55.2 \%, p<0.05)$. The 6 -month culture conversion rate among patients with new onset, one-time TB treatment history or two-time TB treatment history did not show significant differences between the two treatment groups (all $p$ $>0.05)$. 
Table 2

The effect of the type of drug resistance or history of TB treatment on the culture conversion rate at month 6 of the two groups

\begin{tabular}{|c|c|c|c|c|c|}
\hline \multirow[t]{2}{*}{ Indicators } & \multicolumn{2}{|c|}{ Bedaquiline group $(n=102)$} & \multicolumn{2}{|c|}{ Non-bedaquiline group $(n=100)$} & \multirow{2}{*}{$\begin{array}{l}p \\
\text { value }\end{array}$} \\
\hline & Conversion & $\begin{array}{l}\text { Failed to } \\
\text { conversion }\end{array}$ & Conversion & $\begin{array}{l}\text { Failed to } \\
\text { conversion }\end{array}$ & \\
\hline
\end{tabular}

\begin{tabular}{|c|c|c|c|c|c|}
\hline \multicolumn{6}{|l|}{ Type of drug resistance (n, \%) } \\
\hline RR & $3(100.0)$ & $0(0.0)$ & $4(80.0)$ & $1(20.0)$ & 1.000 \\
\hline MDR & $65(87.8)$ & $9(12.2)$ & $50(73.5)$ & $18(26.5)$ & $0.030 *$ \\
\hline XDR & $22(88.0)$ & $3(12.0)$ & $18(66.7)$ & $9(33.3)$ & 0.065 \\
\hline \multicolumn{6}{|l|}{ History of TB treatment $(n, \%)$} \\
\hline New cases & $10(90.9)$ & $1(9.1)$ & $6(75.0)$ & $2(25.0)$ & 0.546 \\
\hline One-time history of TB treatment & 34 (91.9) & $3(8.1)$ & $19(76.0)$ & $6(24.0)$ & 0.169 \\
\hline Two-time history of TB treatment & $27(84.4)$ & $5(15.6)$ & $31(81.6)$ & $7(18.4)$ & 0.757 \\
\hline $\begin{array}{l}\geq \text { Three-time history of TB } \\
\text { treatment }\end{array}$ & $19(86.4)$ & $3(13.6)$ & $16(55.2)$ & $13(44.8)$ & $0.017^{*}$ \\
\hline
\end{tabular}

4. Treatment outcomes

Next, the end-of-treatment outcomes of patients receiving bedaquiline-containing regimens or bedaquiline-free regimens were evaluated. Among the patients in the bedaquiline group, 94 (92.2\%) had a successful TB treatment outcome (cure, $n=71$; treatment completion, $n=23), 3(2.9 \%)$ experienced treatment failure, 4 (3.9\%) were lost to follow-up and $1(1.0 \%)$ died. Besides, treatment success (cure, $n=45$; treatment completion, $n=18$ ), treatment failure, lost to follow-up and death in the non-bedaquiline group were reported in $63(63.0 \%), 27(27.0 \%), 9(9.0 \%)$ and 1 (1.0\%) patients, respectively. Significant differences were found between the two groups $(p<0.001)$. Among the patients who were treated successfully, the median duration of the treatment was 18.0 (IQR, 18.024.0) and 24.0 (IQR, 24.0-24.0) months in the bedaquiline group and non-bedaquiline group, respectively.

As shown in Table 3, the proportions of patients achieving treatment success were significantly different in patients with MDR-TB (93.2\% vs. $69.1 \%, p<0.001)$ and XDR-TB $(88.0 \%$ vs. $51.9 \% ; p<0.05)$ between the two groups. In addition, patients with one-time ( $91.9 \%$ vs. $72.0 \%, p<0.05)$, two-time $(90.6 \%$ vs. $71.1 \%, p<0.001)$ and $\geq$ three-time TB treatment histories $(95.5 \%$ vs. $41.4 \%, p<$ 0.001 ) in the bedaquiline group had higher proportions of treatment success than those in the non-bedaquiline group. 
Table 3

The effect of the type of drug resistance or history of TB treatment on the proportion of treatment success of the two groups

\begin{tabular}{|c|c|c|c|c|c|}
\hline \multirow[t]{2}{*}{ Indicators } & \multicolumn{2}{|c|}{ Bedaquiline group $(n=102)$} & \multicolumn{2}{|c|}{ Non-bedaquiline group $(n=100)$} & \multirow[t]{2}{*}{$p$ value } \\
\hline & Success & Others & Success & Others & \\
\hline \multicolumn{6}{|l|}{ Type of drug resistance $(n, \%)$} \\
\hline RR & $3(100.0)$ & $0(0.0)$ & $2(40.0)$ & $3(60.0)$ & 0.196 \\
\hline MDR & $69(93.2)$ & $5(6.8)$ & $47(69.1)$ & $21(30.9)$ & $<0.001^{*}$ \\
\hline XDR & $22(88.0)$ & $3(12.0)$ & $14(51.9)$ & $13(48.1)$ & $0.005^{*}$ \\
\hline \multicolumn{6}{|l|}{ History of TB treatment $(n, \%)$} \\
\hline New cases & $10(90.9)$ & $1(9.1)$ & $6(75.0)$ & $2(25.0)$ & 0.546 \\
\hline One-time history of TB treatment & $34(91.9)$ & $3(8.1)$ & $18(72.0)$ & $7(28.0)$ & $0.037^{*}$ \\
\hline Two-time history of TB treatment & $29(90.6)$ & $3(9.4)$ & $27(71.1)$ & $11(28.9)$ & $0.041^{*}$ \\
\hline$\geq$ Three-time history of TB treatment & $21(95.5)$ & $1(4.5)$ & $12(41.4)$ & $17(58.6)$ & $<0.001^{*}$ \\
\hline
\end{tabular}

5. Cavity closing rate

The cavity closing rates of patients in the bedaquiline group at month $9(19.6 \%$ vs. $8.0 \%, p=0.017)$ and month $12(39.2 \%$ vs. $15.0 \%$, $p<0.001$ ) were significantly higher than those in the non-bedaquiline group. However, no significant differences were found in the cavity closure rate at month $3(2.9 \%$ vs. $2.0 \%, p=1.000)$ or month $6(11.8 \%$ vs. $4.0 \%, p=0.066)$ between the two groups.

\section{Independent predictor of treatment success and culture reversion}

Univariate and multivariate analyses (Table 4 and Table 5) showed that the use of bedaquiline was an independent predictor of both treatment success ( $\mathrm{OR}=7.356,95 \% \mathrm{Cl}: 2.920-18.530, p<0.001)$ and culture reversion $(\mathrm{OR}=0.124,95 \% \mathrm{Cl}: 0.035-0.452, p<$ 0.001 ) after adjusting for age, sex, cavitary disease, diabetes, TB treatment history, and drug resistance type. 
Table 4

Univariate and multivariate analysis of independent predictors for treatment success

\begin{tabular}{|c|c|c|c|c|c|}
\hline \multirow[t]{2}{*}{ Independent factors } & \multirow{2}{*}{$\begin{array}{l}\text { Treatment } \\
\text { success }\end{array}$} & \multicolumn{2}{|c|}{ Univariate analysis } & \multicolumn{2}{|c|}{ Multivariate analysis } \\
\hline & & OR $(95 \% \mathrm{Cl})$ & $\begin{array}{l}p \\
\text { value }\end{array}$ & OR $(95 \% \mathrm{Cl})$ & $\underset{\text { value }}{p}$ \\
\hline \multicolumn{6}{|l|}{ Age } \\
\hline$<35$ years & $72(85.7)$ & - & - & - & - \\
\hline $35-60$ years & $73(73.0)$ & $\begin{array}{l}0.451(0.212 \\
0.958)\end{array}$ & 0.036 & $\begin{array}{l}2.695(0.640- \\
11.352)\end{array}$ & 0.177 \\
\hline$\geq 60$ years & $14(77.8)$ & $\begin{array}{l}0.583(0.164 \\
2.073)\end{array}$ & 0.629 & $\begin{array}{l}1.003(0.280- \\
3.592)\end{array}$ & 0.997 \\
\hline \multicolumn{6}{|l|}{ Type of drug resistance } \\
\hline $\mathrm{RR}$ & $5(62.5)$ & - & - & & \\
\hline MDR & $116(76.3)$ & $\begin{array}{l}0.831(0.182- \\
3.790)\end{array}$ & 0.811 & & \\
\hline XDR & $36(58.1)$ & $\begin{array}{l}2.677(0.601- \\
11.916)\end{array}$ & 0.181 & & \\
\hline Gender (male/female) & $\begin{array}{l}114(77.0) / 42 \\
(79.2)\end{array}$ & $\begin{array}{l}0.878(0.408- \\
1.890)\end{array}$ & 0.756 & & \\
\hline $\begin{array}{l}\text { History of TB treatment (new/previously } \\
\text { treated) }\end{array}$ & $\begin{array}{l}16(84.2) / 141 \\
(77.0)\end{array}$ & $\begin{array}{l}1.589(0.442- \\
5.716)\end{array}$ & 0.475 & & \\
\hline Cavitary disease at baseline (yes/no) & $\begin{array}{l}114(81.4) / 39 \\
(72.2)\end{array}$ & $\begin{array}{l}0.593(0.285- \\
1.233)\end{array}$ & 0.159 & & \\
\hline Concomitant diabetes (yes/no) & $\begin{array}{l}21(72.4) / 136 \\
(78.6)\end{array}$ & $\begin{array}{l}1.400(0.574- \\
3.416)\end{array}$ & 0.458 & & \\
\hline $\begin{array}{l}\text { Treatment regimen (bedaquiline/non- } \\
\text { bedaquiline) }\end{array}$ & $\begin{array}{l}94(92.2) / 63 \\
(63.0)\end{array}$ & $\begin{array}{l}6.901(3.015- \\
15.797)\end{array}$ & $\begin{array}{l}< \\
0.001^{*}\end{array}$ & $\begin{array}{l}7.356(2.920- \\
18.530)\end{array}$ & $\dot{0.001}$ \\
\hline
\end{tabular}


Table 5

Univariate and multivariate analysis of independent predictors for reversion to positive culture

\begin{tabular}{|c|c|c|c|c|c|}
\hline \multirow[t]{2}{*}{ Independent factors } & \multirow{2}{*}{$\begin{array}{l}\text { Reversion to positive } \\
\text { culture }\end{array}$} & \multicolumn{2}{|c|}{ Univariate analysis } & \multicolumn{2}{|c|}{ Multivariate analysis } \\
\hline & & OR $(95 \% \mathrm{Cl})$ & $\begin{array}{l}p \\
\text { value }\end{array}$ & OR $(95 \% \mathrm{Cl})$ & $\begin{array}{l}p \\
\text { value }\end{array}$ \\
\hline \multicolumn{6}{|l|}{ Age } \\
\hline$<35$ years & $5(6.0)$ & - & - & & \\
\hline $35-60$ years & $15(15.0)$ & $\begin{array}{l}2.788(0.968- \\
8.027)\end{array}$ & 0.050 & & \\
\hline$\geq 60$ years & $1(5.6)$ & $\begin{array}{l}0.929(0.102- \\
8.473)\end{array}$ & 0.94 & & \\
\hline \multicolumn{6}{|l|}{ Type of drug resistance } \\
\hline RR & $1(12.5)$ & - & - & & \\
\hline MDR & $11(7.7)$ & $\begin{array}{l}0.558(0.066- \\
5.219)\end{array}$ & 0.630 & & \\
\hline XDR & 9 (17.3) & $\begin{array}{l}1.465(0.160- \\
13.423)\end{array}$ & 0.734 & & \\
\hline $\begin{array}{l}\text { History of TB treatment } \\
\text { (new/previously treated) }\end{array}$ & $2(10.5) / 19(10.4)$ & $\begin{array}{l}1.015(0.218- \\
4.738)\end{array}$ & 0.984 & & \\
\hline Cavitary disease at baseline (yes/no) & $18(12.9) / 3(5.6)$ & $\begin{array}{l}0.399(0.113- \\
1.413)\end{array}$ & 0.142 & & \\
\hline Concomitant diabetes (yes/no) & $3(10.3) / 18$ (10.4) & $\begin{array}{l}1.006(0.277- \\
3.660)\end{array}$ & 0.992 & & \\
\hline Gender (male/female) & $20(13.5) / 1(1.9)$ & $\begin{array}{l}8.125(1.063- \\
62.116)\end{array}$ & 0.018 & $\begin{array}{l}1.254(0.386- \\
4.082)\end{array}$ & 0.705 \\
\hline $\begin{array}{l}\text { Treatment regimen (bedaquiline/non- } \\
\text { bedaquiline) }\end{array}$ & $3(2.9) / 18(18.0)$ & $\begin{array}{l}0.138(0.039- \\
0.485)\end{array}$ & $<.001^{*}$ & $\begin{array}{l}0.124(0.035- \\
0.452)\end{array}$ & 0.002 \\
\hline
\end{tabular}

\section{AEs}

In the bedaquiline group, 27 patients $(26.5 \%)$ reported a total of 34 AEs during the total treatment duration. The most commonly reported AEs were nephrotoxicity $(n=12,11.8 \%)$, hepatotoxicity $(n=9,8.8 \%)$, peripheral neuropathy $(n=3,3.0 \%)$, leukopenia $(n=2$, $2.0 \%)$, hypokalemia $(n=2,2.0 \%)$, ototoxicity $(n=2,2.0 \%)$, and others $(n=4,3.9 \%)$. No QTcF interval prolongation was observed in any of 102 patients who received the bedaquiline-containing regimens (Table 6). 
Table 6

Summary of adverse events in the two groups

\begin{tabular}{|c|c|c|c|c|c|c|c|c|}
\hline Groups & $\begin{array}{l}\text { Nephrotoxicity } \\
(n, \%)\end{array}$ & $\begin{array}{l}\text { Hepatotoxicity } \\
(n, \%)\end{array}$ & $\begin{array}{l}\text { Peripheral } \\
\text { neuropathy } \\
(n, \%)\end{array}$ & $\begin{array}{l}\text { Leukopenia } \\
(n, \%)\end{array}$ & $\begin{array}{l}\text { Hypokalemia } \\
(n, \%)\end{array}$ & $\begin{array}{l}\text { Ototoxicity } \\
(n, \%)\end{array}$ & $\begin{array}{l}\text { Others } \\
(n, \%)\end{array}$ & $\underset{\text { value }}{p}$ \\
\hline $\begin{array}{l}\text { Bedaquiline } \\
\text { group }(n= \\
\text { 102) }\end{array}$ & $12(11.8)$ & $9(8.8)$ & $3(3.0)$ & $2(2.0)$ & $2(2.0)$ & $2(2.0)$ & $4(3.9)$ & 0.206 \\
\hline $\begin{array}{l}\text { Non- } \\
\text { bedaquiline } \\
\text { group }(n= \\
100)\end{array}$ & $3(3.0)$ & $2(2.0)$ & $1(1.0)$ & $2(2.0)$ & $1(1.0)$ & $8(8.0)$ & $7(7.0)$ & \\
\hline $\mathrm{n}$, number & & & & & & & & \\
\hline
\end{tabular}

In the non-bedaquiline group, a total of 24 AEs were observed in 19 patients (19.0\%). The most frequently observed AEs were ototoxicity $(n=8,8.0 \%)$, nephrotoxicity $(n=3,3.0 \%)$, hepatotoxicity $(n=2,2.0 \%)$, leukopenia $(n=2,2.0 \%)$, hypokalemia $(n=1,1.0 \%)$, peripheral neuropathy $(n=1,1.0 \%)$, and others $(n=7,7.0 \%)$.

Significant differences were not observed in the occurrence of AEs reported in these two groups $(p=0.206)$. In addition, no serious AEs were observed in any patients from the two groups.

\section{Differences in treatment outcomes among different regimens}

Subsequently, the end-of-treatment outcomes and 6-month culture conversion rates of the patients receiving an all-oral bedaquilinebased regimen, injectable-containing but bedaquiline-free regimen, or injectable and bedaquiline-containing regimen were evaluated. As shown in Fig. 3A and 3B, significant differences were observed between the three treatment groups in the proportions of end-of-treatment success $(89.8 \%$ vs. $65.5 \%$ vs. $95.3 \%, p<0.001)$ and the 6 -month culture conversion rate $(86.4 \%$ vs. $72.9 \%$ vs. $90.7 \%, p=0.024)$.

\section{Discussion}

The End TB strategy of the WHO aims to end the global TB epidemic by 2035 [12]. However, compared to drug-susceptible TB, current treatment outcomes for DR-TB are relatively poor, especially for patients with refractory RR/MDR-TB [13]. Moreover, treatment options for refractory RR/MDR-TB are extremely limited. Patients often experience failed DR-TB treatment and present complicated medical histories of second-line drugs or intolerance to several effective drugs; therefore, treatment regimens often rely on a set of drugs with a high pill burden, poorly established efficacy and severe AE profiles [2]. In some settings, poor efficacy and tolerability might lead to the discontinuation of treatment, which increases patient mortality and transmission risk of highly resistant strains [14]. Therefore, effective and safe treatment regimens for refractory RR/MDR-TB must be developed for the realization of the WHO's End TB strategy.

Bedaquiline is an ATP synthase inhibitor and a novel agent recommended by the WHO for the treatment of MDR-TB [15]. Adding bedaquiline to standard treatment regimens for MDR-TB has been shown to improve the long-term survival of patients, reduce the time for sputum culture conversion, and increase the proportion of patients with consecutive negative culture results [16-18]. Given that patients with refractory RR/MDR-TB have few treatment options, bedaquiline might represent a useful choice for this population. However, evidence about the effectiveness and safety of bedaquiline-containing regimens in this group of patients is lacking. Therefore, we conducted a retrospective cohort study and compared the treatment outcomes of bedaquiline-containing and bedaquiline-free regimens for the treatment of patients with refractory RR/MDR-TB in East China.

In previous studies, the culture conversion rate at month 6 in patients treated with bedaquiline-containing regimens varied from $64-100 \%$ and the treatment success rate ranged between $52 \%$ and $85 \%$ [18]. Both outcomes were higher in patients treated with bedaquiline than in those without bedaquiline. Similarly, patients receiving bedaquiline-containing regimens in the present study had higher culture conversion rates at month $6(90.2 \%$ vs. $72.0 \%)$ and treatment success rates $(92.2 \%$ vs. $63.0 \%)$ than those receiving bedaquiline-free regimens. Moreover, the use of bedaquiline was identified as an independent predictor of treatment 
success (OR $=7.356,95 \% \mathrm{Cl}: 2.920-18.530, p<0.001)$, thus reflecting the superior antibacterial and bactericidal activity of bedaquiline against tubercle bacilli [19]. Interestingly, the treatment success rate observed in our patients treated with bedaquiline was slightly higher than that in previous reports, which may be related to the background drugs included in the bedaquilinecontaining regimens. Nearly all participants in the bedaquiline group received pyrazinamide (100\%), linezolid (90.2\%), and clofazimine (90.2\%). Evidence has shown that linezolid has a positive effect on the clinical outcomes of patients with DR-TB [20, 21]. The addition of pyrazinamide to bedaquiline-clofazimine combination regimens resulted in even more rapid sterilizing activity $[22,23]$. Furthermore, cautious management and strict patient monitoring might also contribute to the high rate of treatment success. Taken together, the inclusion of bedaquiline in the treatment regimens for patients with refractory RR/MDR-TB was associated with good outcomes.

To the best of our knowledge, this study showed for the first time that patients with a $\geq$ one-time TB treatment history, MDR-TB or XDR-TB were more likely to benefit from bedaquiline-containing regimens and showed a higher treatment success rate than those receiving bedaquiline-free regimens. The frequency of drug resistance for anti-TB drugs of previously treated TB was found to be five times higher than that of new-onset patients [24]. An increase in resistance to anti-TB drugs against Mycobacterium tuberculosis poses a major threat to TB control [25]. In addition, compared with RR-TB, MDR-TB and XDR-TB were more difficult to manage and showed broad-spectrum resistance, poor outcomes and high mortality [26]. Overall, these findings further confirmed that bedaquiline-containing regimens showed superior efficacy in treatment patients with refractory and complicated DR-TB.

Another important finding from this study was that the end-of-treatment success proportion and 6-month culture conversion rate varied with the treatment regimen. Overall, there was a high treatment success rate and culture conversion rate for patients who received bedaquiline, regardless of whether an injectable agent was administered. Prior to August 2018, RR/MDR-TB treatment guidelines recommended parenteral administration of capreomycin or aminoglycoside (kanamycin or amikacin) as part of the treatment [27]. However, due to the significant toxicity and patient intolerance that led to treatment interruption [28], the WHO deprioritized the injectable-containing regimens in 2019 and recommended the use of all-oral regimens [27]. Therefore, although the treatment success rate and culture conversion rate in the present study were slightly higher in patients treated with bedaquiline and injectable agents than in those who did not receive injectable agents, an all-oral bedaquiline-based regimen was recommended for the treatment of patients with refractory RR/MDR-TB.

Of special concern was that the use of bedaquiline was associated with QTCF interval prolongation [29]. In our cohort, no patient experienced QTcF interval prolongation, suggesting that bedaquiline, even in combination with clofazimine, might be safe. Other studies have reported a similarly low rate of cardiotoxicity [30,31]. This finding could therefore alleviate some concerns about the risk of cardiotoxicity associated with the use of bedaquiline.

Several limitations were observed in this study. First, this retrospective cohort study included patients receiving bedaquilinecontaining regimens from August 2018 to August 2020 and patients receiving bedaquiline-free regimens from August 2016 to July 2018. Although the baseline characteristics of patients were well balanced between the two groups, the implementation conditions (monitoring, quality of care, staffing) changed over time, which might cause bias in the analyses. Second, AEs were based on selfreports and clinician notes from handwritten medical records, which is a limitation inherent in all retrospective studies. The overall low rate of AEs observed in our study could be a consequence of underreporting. However, despite the combined use of other drugs that also cause QTcF interval prolongation, the current data showed the absence of critical cardiac AEs in patients receiving bedaquiline, thus demonstrating the safety of bedaquiline under procedural conditions for treating patients with refractory RR/MDR-TB. Therefore, it is necessary to conduct prospective studies to comprehensively and accurately evaluate the effectiveness and safety of bedaquiline-containing regimens for the treatment of refractory RR/MDR-TB.

\section{Conclusions}

Bedaquiline-containing regimens resulted in better treatment outcomes and comparable safety compared to bedaquiline-free regimens for patients with refractory pulmonary RR/MDR-TB. The use of bedaquiline was identified as an independent predictor of favorable treatment outcomes. These findings provide significant positive insights in support of the recent WHO recommendations on the wide use of bedaquiline. 


\section{Declarations \\ Declarations}

\section{Ethics approval and consent to participate:}

This study was approved by the ethics committee of Shanghai Pulmonary Hospital (number 114JH). Written informed consent was obtained from all participants and their guardians.

\section{Consent for publication:}

Not applicable.

\section{Declarations}

\section{Declaration of Conflicting Interests}

The authors declare that they have no conflicts of interest.

\section{Funding:}

This work was supported by the 13th Five-Year National Science and Technology Major Project for Infectious Diseases (2018ZX10725-509 and 2018ZX10722301-001) and Shanghai Pulmonary Hospital fund (FK1945 and FKLY20017).

\section{Authors' Contributions:}

Conception and design: LF, ZMZ and YL; Collection of data: SZ; Analysis and interpretation of data: YS; Provision of materials and samples: YY and ZSZ; Drafting of the article: SZ; Approval of the article: LF; Administrative support: ZZ. All authors have read and approved the final manuscript.

\section{Acknowledgment:}

None.

\section{References}

1. Mirnejad R, Asadi A, Khoshnood S, et al. Clofazimine: A useful antibiotic for drug-resistant tuberculosis. Biomedicine \& pharmacotherapy $=$ Biomedecine $\&$ pharmacotherapie 2018;105:1353-1359

2. Global tuberculosis report 2020. Geneva: World Health Organization 2020: Licence: CC BY-NC-SA 3.0 IGO

3. Ahmad N, Ahuja SD, Akkerman OW, et al. Treatment correlates of successful outcomes in pulmonary multidrug-resistant tuberculosis: an individual patient data meta-analysis. Lancet (London, England) 2018;392:821-834

4. Pym AS, Diacon AH, Tang SJ, et al. Bedaquiline in the treatment of multidrug- and extensively drug-resistant tuberculosis. The European respiratory journal 2016;47:564-574

5. WHO consolidated guidelines on tuberculosis: Module 4: Treatment - Drug-resistant tuberculosis treatment. In. Geneva: World Health Organization; 2020:Licence: CC BY-NC-SA 3.0 IGO.

6. Olayanju O, Limberis J, Esmail A, et al. Long-term bedaquiline-related treatment outcomes in patients with extensively drugresistant tuberculosis from South Africa. The European respiratory journal 2018;51

7. Olayanju O, Esmail A, Limberis J, Dheda K. A regimen containing bedaquiline and delamanid compared to bedaquiline in patients with drug-resistant tuberculosis. The European respiratory journal 2020;55 
8. Agnarson AM, Wang XC, Potluri R, et al. Long-term impact of the adoption of bedaquiline-containing regimens on the burden of drug-resistant tuberculosis in China. BMC infectious diseases 2020;20:113

9. Pietersen $\mathrm{E}$, Ignatius $\mathrm{E}$, Streicher EM, et al. Long-term outcomes of patients with extensively drug-resistant tuberculosis in South Africa: a cohort study. Lancet (London, England) 2014;383:1230-1239

10. Definitions and reporting framework for tuberculosis-2013 revision: updated December 2014 and January 2020. Geneva: World Health Organization 2013: Licence: CC BY-NC-SA 3.0 IGO

11. Guideline IHT. Clinical safety data management: definitions and standards for expedited reporting E2A. In, International conference on harmonisation of technical requirements for registration of pharmaceuticals for human use; 1994

12. Uplekar M, Weil D, Lonnroth K, et al. WHO's new end TB strategy. Lancet (London, England) 2015;385:1799-1801

13. Ndjeka N, Schnippel K, Master I, et al. High treatment success rate for multidrug-resistant and extensively drug-resistant tuberculosis using a bedaquiline-containing treatment regimen. The European respiratory journal 2018;52

14. Cox H, Ford N. Linezolid for the treatment of complicated drug-resistant tuberculosis: a systematic review and meta-analysis. The international journal of tuberculosis and lung disease: the official journal of the International Union against Tuberculosis and Lung Disease 2012;16:447-454

15. Ismail NA, Omar SV, Joseph L, et al. Defining Bedaquiline Susceptibility, Resistance, Cross-Resistance and Associated Genetic Determinants: A Retrospective Cohort Study. EBioMedicine 2018;28:136-142

16. Schnippel K, Ndjeka N, Maartens G, et al. Effect of bedaquiline on mortality in South African patients with drug-resistant tuberculosis: a retrospective cohort study. The Lancet Respiratory medicine 2018;6:699-706

17. Shi L, Gao J, Gao M, et al. Interim Effectiveness and Safety Comparison of Bedaquiline-Containing Regimens for Treatment of Diabetic Versus Non-Diabetic MDR/XDR-TB Patients in China: A Multicenter Retrospective Cohort Study. Infectious diseases and therapy $2021 ; 10: 457-470$

18. Chesov D, Heyckendorf J, Alexandru S, et al. Impact of bedaquiline on treatment outcomes of multidrug-resistant tuberculosis in a high-burden country. The European respiratory journal 2021;57

19. Gao M, Gao J, Xie L, et al. Early outcome and safety of bedaquiline-containing regimens for treatment of MDR-and XDR-TB in China: a multicentre study. Clinical microbiology and infection: the official publication of the European Society of Clinical Microbiology and Infectious Diseases 2021;27:597-602

20. Lee M, Lee J, Carroll MW, et al. Linezolid for treatment of chronic extensively drug-resistant tuberculosis. The New England journal of medicine 2012;367:1508-1518

21. Zhang L, Pang Y, Yu X, et al. Linezolid in the treatment of extensively drug-resistant tuberculosis. Infection 2014;42:705-711

22. Williams K, Minkowski A, Amoabeng $\mathrm{O}$, et al. Sterilizing activities of novel combinations lacking first- and second-line drugs in a murine model of tuberculosis. Antimicrobial agents and chemotherapy 2012;56:3114-3120

23. Xu J, Tasneen R, Peloquin CA, et al. Verapamil Increases the Bioavailability and Efficacy of Bedaquiline but Not Clofazimine in a Murine Model of Tuberculosis. Antimicrobial agents and chemotherapy 2018;62

24. Ullah I, Javaid A, Tahir Z, et al. Pattern of Drug Resistance and Risk Factors Associated with Development of Drug Resistant Mycobacterium tuberculosis in Pakistan. PLoS One 2016;11:e0147529

25. Donald PR, van Helden PD. The global burden of tuberculosis-combating drug resistance in difficult times. The New England journal of medicine 2009;360:2393-2395

26. Migliori GB, Tiberi S, Zumla A, et al. MDR/XDR-TB management of patients and contacts: Challenges facing the new decade. The 2020 clinical update by the Global Tuberculosis Network. International journal of infectious diseases: IJID : official publication of the International Society for Infectious Diseases 2020;92s:S15-s25

27. WHO consolidated guidelines on drug-resistant tuberculosis treatment. In. Geneva: World Health Organization; 2019. License: CC BY-NC-SA 3.0 IGO

28. Khan PY, Franke MF, Hewison C, et al. All-oral longer regimens are effective for the management of multidrug resistant tuberculosis in high burden settings. The European respiratory journal 2021

29. Mase S, Chorba T, Parks S, et al. Bedaquiline for the Treatment of Multidrug-resistant Tuberculosis in the United States. Clinical infectious diseases: an official publication of the Infectious Diseases Society of America 2020;71:1010-1016

Page $12 / 14$ 
30. Guglielmetti L, Tiberi S, Burman M, et al. QT prolongation and cardiac toxicity of new tuberculosis drugs in Europe: a Tuberculosis Network European Trialsgroup (TBnet) study. The European respiratory journal 2018;52

31. Mbuagbaw L, Guglielmetti L, Hewison C, et al. Outcomes of Bedaquiline Treatment in Patients with Multidrug-Resistant Tuberculosis. Emerging infectious diseases 2019;25:936-943

\section{Figures}

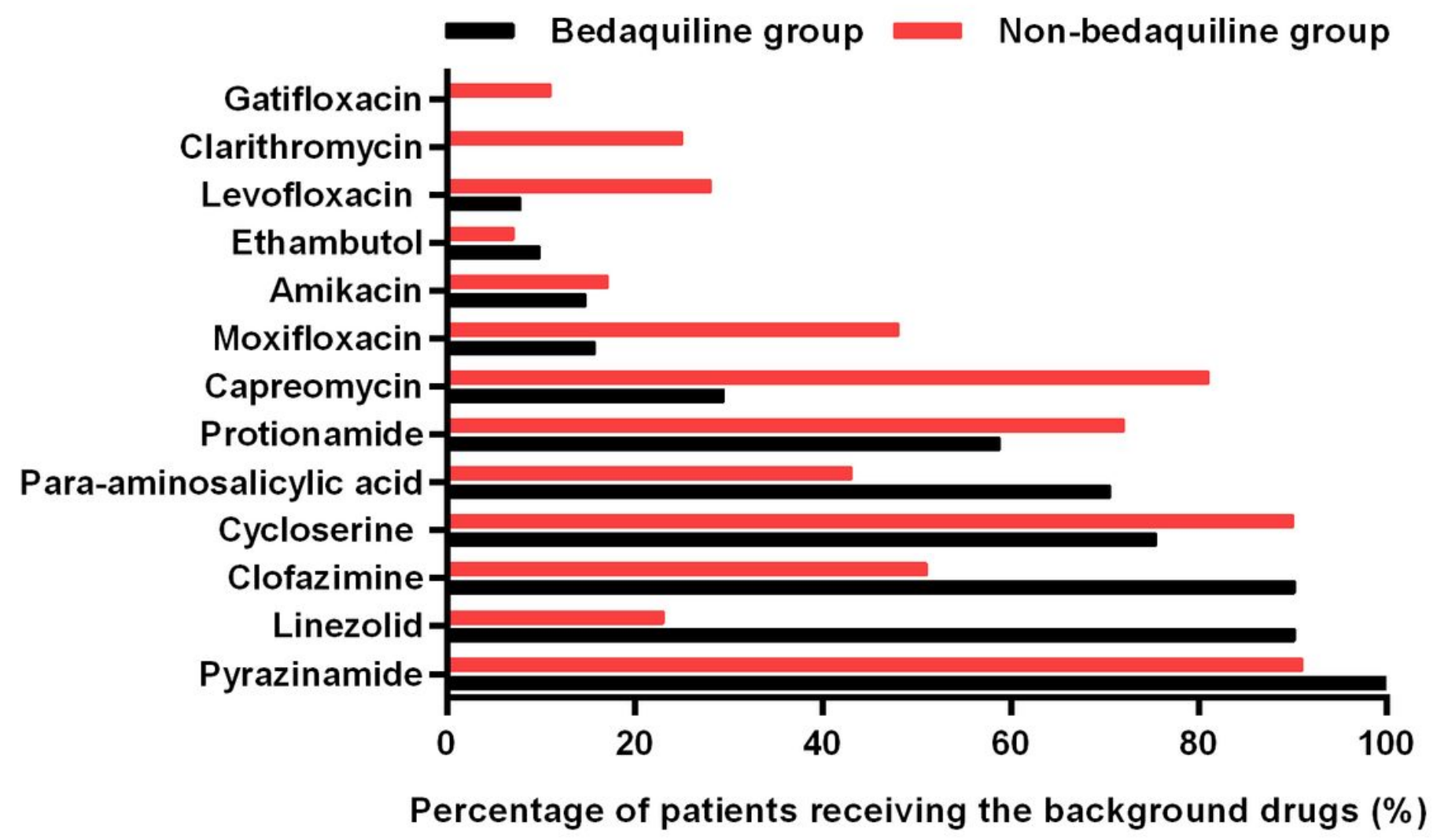

Figure 1

Background drugs included in the treatment regimens of patients from the two groups
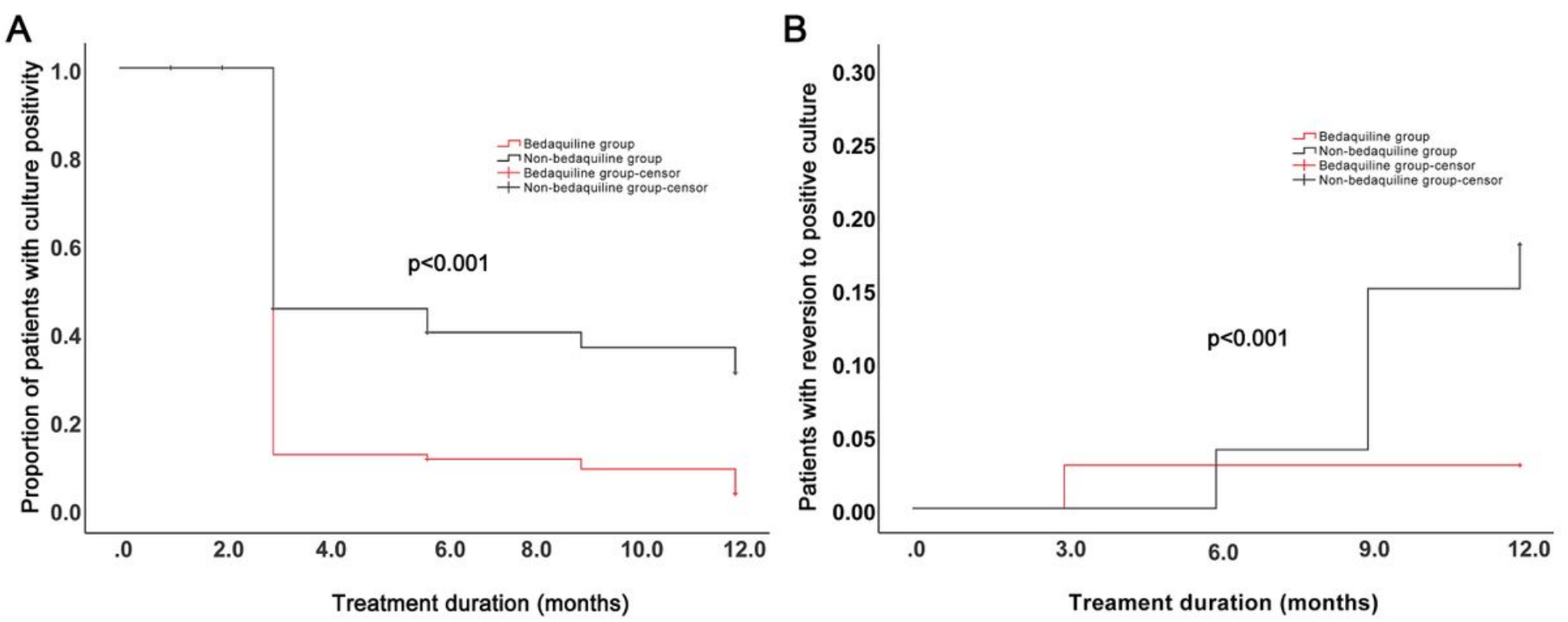
Figure 2

Sputum culture conversion of patients receiving bedaquiline-containing and bedaquiline-free regimens A, Kaplan-Meier survival curves for sputum culture conversion. B, Kaplan-Meier survival curves for culture reversion.
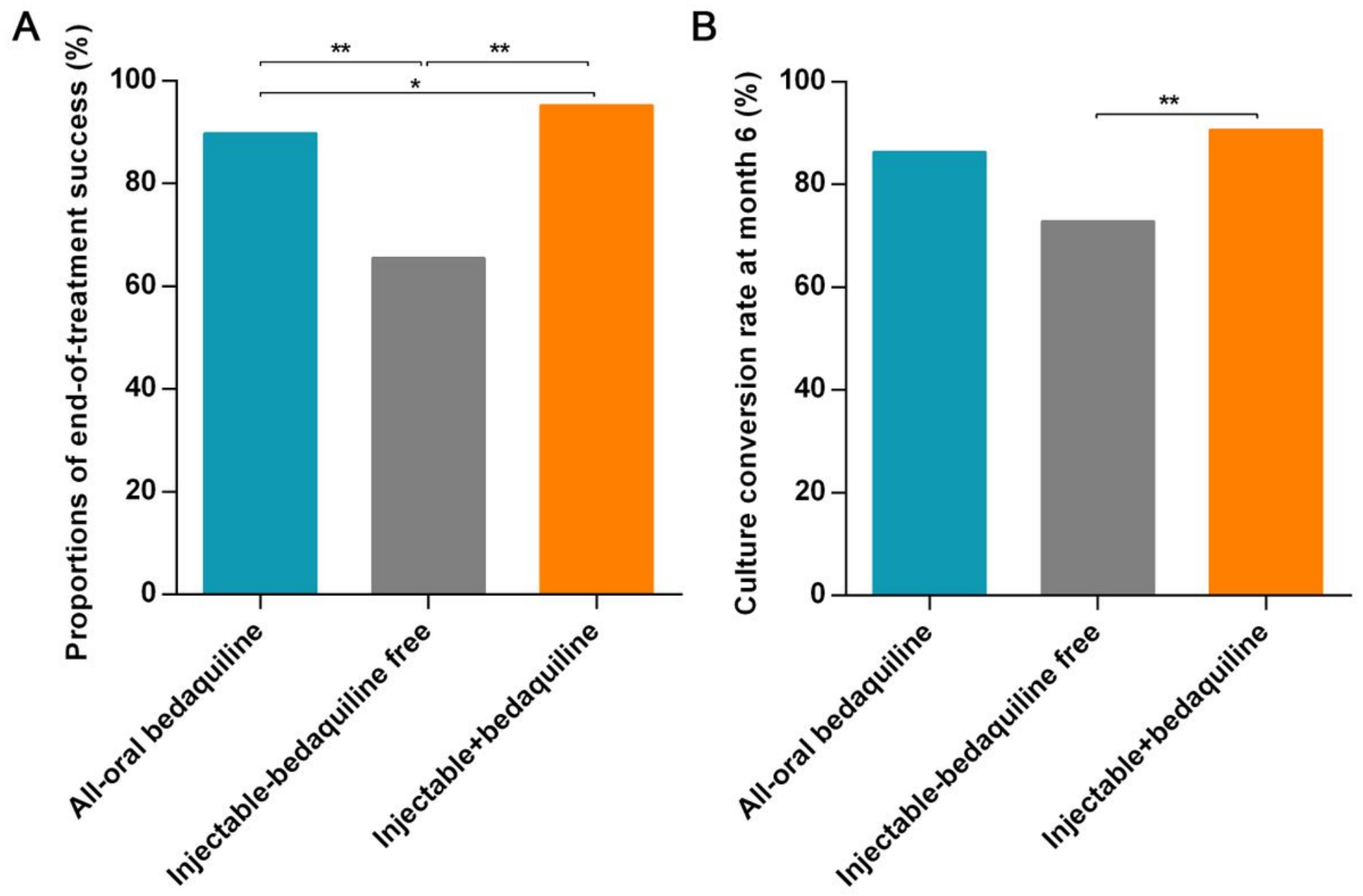

Figure 3

Treatment outcomes of patients receiving different regimens A, Proportions of end-of-treatment success of patients receiving different regimens. $B$, Culture conversion rate at month 6 of patients receiving different regimens. ${ }^{*}, p<0.05 ; * \star, p<0.01$

\section{Supplementary Files}

This is a list of supplementary files associated with this preprint. Click to download.

- Table1.docx 